\title{
Optical Imaging Modalities: Principles and Applications in Preclinical Research and Clinical Settings
}

\author{
Giacomo Pirovano ${ }^{1}$, Sheryl Roberts ${ }^{1}$, Susanne Kossatz ${ }^{2-4}$, and Thomas Reiner ${ }^{1,5,6}$ \\ ${ }^{1}$ Department of Radiology, Memorial Sloan Kettering Cancer Center, New York, New York; ${ }^{2}$ Department of Nuclear Medicine, \\ University Hospital Klinikum Rechts der Isar, Technical University Munich, Munich, Germany; ${ }^{3}$ Central Institute for Translational \\ Cancer Research, Technical University of Munich, Munich, Germany; ${ }^{4}$ Department of Chemistry, Technical University of Munich, \\ Munich, Germany; ${ }^{5}$ Department of Radiology, Weill Cornell Medical College, New York, New York; and ${ }^{6}$ Chemical Biology Program, \\ Memorial Sloan Kettering Cancer Center, New York, New York
}

\begin{abstract}
Learning Objectives: On successful completion of this activity, participants should be able to (1) compare and judge the most appropriate imaging technique based on disease nature; (2) apply excitation source and appropriate readout depending on imaging techniques; and (3) recognize and interpret published molecular imaging data.
\end{abstract}

Financial Disclosure: Drs. Kossatz and Reiner are shareholders of Summit Biomedical Imaging, LLC, and coinventors on a filed U.S. patent (WO2016164771) held by MSK that covers methods of use for PARPi-FL. Dr. Reiner is also a coinventor on a U.S. patent (WO2012074840) held by the General Hospital Corporation that covers the composition of PARPi-FL; is a coinventor on a U.S. patent (WO2016033293) held by MSK that covers methods for the synthesis and use of ${ }^{18} \mathrm{~F}-\mathrm{PARPi},{ }^{131} \mathrm{I}-\mathrm{PARPi}$, and ${ }^{123} \mathrm{I}-\mathrm{MAP}$; and is a paid consultant for Theragnostics, Inc. The authors of this article have indicated no other relevant relationships that could be perceived as a real or apparent conflict of interest.

CME Credit: SNMMI is accredited by the Accreditation Council for Continuing Medical Education (ACCME) to sponsor continuing education for physicians. SNMMI designates each JNM continuing education article for a maximum of 2.0 AMA PRA Category 1 Credits. Physicians should claim only credit commensurate with the extent of their participation in the activity. For CE credit, SAM, and other credit types, participants can access this activity through the SNMMI website (http://www.snmmilearningcenter.org) through October 2023.

With the ability to noninvasively image and monitor molecular processes within tumors, molecular imaging represents a fundamental tool for cancer scientists. In the current review, we describe emergent optical technologies for molecular imaging. We aim to provide the reader with an overview of the fundamental principles on which each imaging strategy is based, to introduce established and future applications, and to provide a rationale for selecting optical technologies for molecular imaging depending on disease location, biology, and anatomy. To accelerate clinical translation of imaging techniques, we also describe examples of practical applications in patients. Elevating these techniques into standardof-care tools will transform patient stratification, disease monitoring, and response evaluation.

Key Words: imaging; optical modalities; cancer

J Nucl Med 2020; 61:1419-1427

DOI: 10.2967/jnumed.119.238279

$\mathbf{O}$

ptical technologies for molecular imaging enable the noninvasive visualization, characterization, and often quantification of structures and biologic processes at the cellular and molecular level in clinical settings. One of the aims of molecular imaging is the application of laboratory tools and methods to an in vivo scenario where the ability to distinguish anatomic and molecular structures is of particular importance for both early diagnosis and

Received Mar. 27, 2020; revision accepted Jun. 30, 2020

For correspondence or reprints contact: Thomas Reiner, Memorial Sloan Kettering Cancer Center, 1275 York Ave., New York, NY 10021.

E-mail: reinert@mskcc.org

Published online Aug. 6, 2020.

COPYRIGHT (c) 2020 by the Society of Nuclear Medicine and Molecular Imaging. therapeutic intervention. Nontoxic and noninvasive imaging methods allow clinicians and researchers to analyze intact organisms over time without needing to remove or permanently alter the observed tissues and thus to study cells and tissue in their original location without influencing interactions with the microenvironment. Further, with real-time imaging, dynamic studies of target biology can provide a more informative picture. This is of particular importance for the investigation of diseases such as cancer (1). Typically, optical imaging requires the injection of an imaging probe able to produce a detectable and targeted signal. A successful imaging probe must have a favorable pharmacokinetic profile, with the ability to access a target molecule with high affinity after passing through the biologic barriers and membranes that separate the target from the bloodstream. Simultaneously, the probe must be detectable with high sensitivity, quickly, and at high resolution. Depending on the applications, molecular imaging technologies must also integrate into existing environments and workflows (e.g., suitable size, weight, and comfort) to have a chance for clinical translation. In this work, we discuss the imaging techniques fluorescence imaging, bioluminescence imaging (BLI), optoacoustic imaging, and Cerenkov luminescence (CL). These molecular imaging techniques have distinct origins and histories; they differ in their operational complexity for the user, in their sensitivity, and in whether they depend on external contrast agents or generate their signals from intrinsic molecular signatures and processes (Fig. 1). Contrast agents have been introduced for molecular imaging applications and for improved readouts. A contrast agent is an additional substance that can be paired to the imaging technique in order to increase the target-to-background signal and therefore produce clearer images. In most cases, the principle or technology underlying a given technique has been known for decades or longer, even when it only recently became a true molecular imaging modality. For example, fluorescence was first described in a scientific publication in 1852 by George 


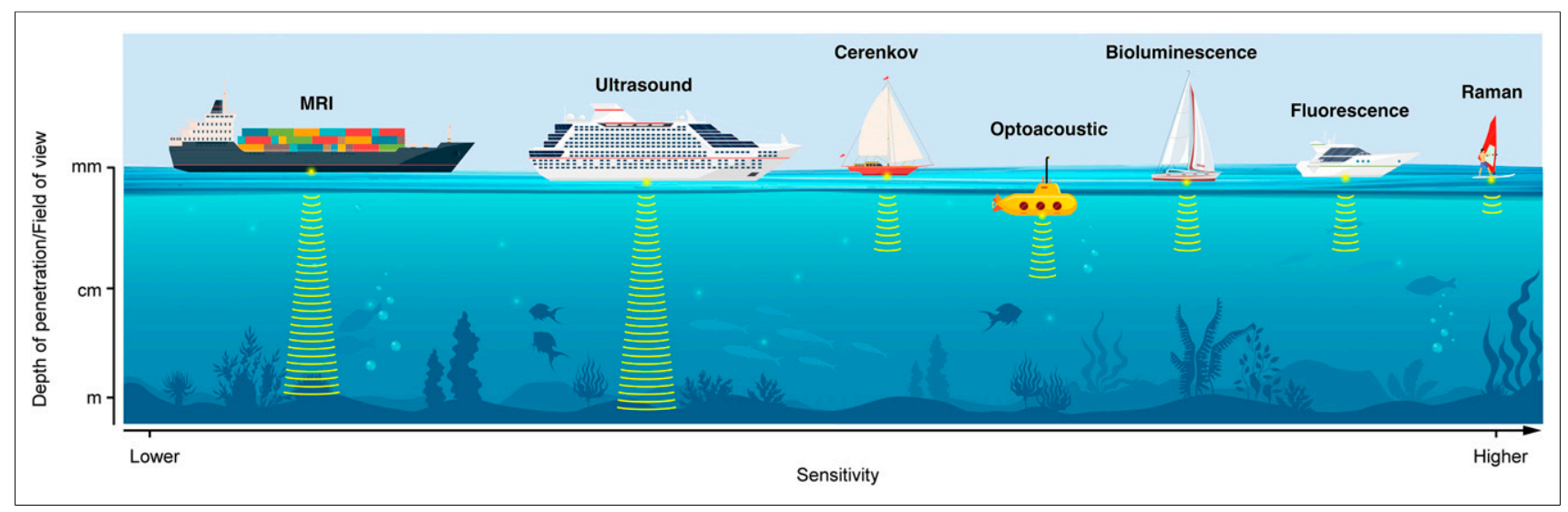

FIGURE 1. Schematic representation of various optical imaging modalities discussed in this review. Different modalities are represented as different boats, each with its individual pros and cons and its specific utility. (Printed with permission of Memorial Sloan Kettering Cancer Center.)

Gabriel Stokes, opening the door to the development of a large set of fluorescent dyes and the invention of the fluorescent microscope. Fluorescent dyes began to be used for clinical applications in vitro and in vivo. The first publication on bioluminescence and chemiluminescence, by Conrad Gesner, dates back to 1555; its 2 main components, luciferin and luciferase, were identified in 1956. CL was studied in 1934 by Pavel Cerenkov, whereas the first CL images for clinical applications date to 2009 , after ${ }^{18} \mathrm{~F}-$ FDG injection. CL can be detected in the dark and has potential to image lesions in patients after injection of radioactive material. The same can be said of the rapidly growing field of optoacoustic imaging; this technology is based on excitation with light using a nanosecond-pulsed laser followed by the detection of emitted ultrasound. Each of the techniques described here has distinct advantages and disadvantages, and the choice between them should be based on the variable settings present in the study of interest.

\section{FLUORESCENCE IMAGING}

Fluorescence microscopy techniques are used to increase image contrast and spatial resolution-an integral part of modern cell biology that is rapidly evolving in both preclinical and clinical settings, with new techniques, contrast agents, and equipment appearing almost every day (2). The wide variety of available fluorescent microscopes-wide-field, laser scanning confocal, 2photon, scanning disk confocal, total internal reflection, super resolution-combine with methods such as stimulated emission depletion, structured illumination, Förster resonance energy transfer, fluorescence lifetime imaging, fluorescence recovery after photobleaching, and fluorescence correlation spectroscopy (2). Fluorescence microscopy can be used for analytic chemistry, spectroscopy, biosensing, fluorescence-activated cell sorting, DNA detection, flow cytometry, live cell imaging, fluorescent proteins, labeling, and fluorescence image-guided surgeries (3). In preclinical settings, the role of fluorescence imaging in different phases of drug development is significant for studying drug-mediated protein-protein interactions in cell culture and in vivo (4). Druginduced protein interactions can be imaged and quantified in vitro or in cell culture. This allows for high-throughput content screening of compounds, which includes, but is not limited to, mechanistic studies, elucidation of molecular function, biologics drug discovery, and toxicology. The strategy is extended to image drug effects on tumor proliferation, tumor apoptosis, and tumor angiogenesis (5). In clinical settings, robotic surgeries are implemented with vision systems based on fluorescent cameras; this is the case with the da Vinci (Intuitive Surgical), Artemis (innoMedicus), and Stryker fluorescence imaging vision systems (6).

\section{Principles of Fluorescence Imaging}

The fluorescent process comprises the absorption of light, in tissues from an array of light sources, followed by the emission of some of this light a few nanoseconds later (Fig. 2A), which is captured with an array of detectors. The aim is to separate the emitted light from the excitation light. A light propagation model based on the distribution of scattering and absorption parameters is used and compared with the experimental intensity measurements of all the source detectors (7). Therefore, the evolution of optical filters, filter cubes, dichroic mirrors, and their orientation to one another is key to successful imaging with respect to contrast agents. There are thousands of exogenous fluorescent probes that provide the means of labeling many aspects of biologic systems, from small-molecule, peptide-based, and affibody-based (Affibody $\mathrm{AB})$ to substrate- and activity-based, as described in detail in Koch and Ntziachristos (8). Recent advances have been made in the development of probes in the near-infrared (NIR) window, in maximum quantum yield efficiency, in large stoke shifts, and in overcoming bleaching. In addition, since the discovery of green fluorescent proteins (GFP) from aequorin, fluorescent proteins have been widely genetically encoded and adapted in a wide range of applications from neuroscience to cancer imaging (9).

\section{Clinical Advances}

Radical resection during surgery is the preferred treatment for most cancers. Therefore, practical methods for augmenting the ability to resect tumors are desired, as well as methods to visualize and avoid compromising the surrounding nerves or tissue. Information on the tumor and on vital structures is crucial to surgical success. Thus, adopting imaging camera prototypes in combination with the relevant fluorescent dye is essential. Such examples as intraoperative image-guided transoral robotic surgery, fluorescence cystoscopy, and the multispectral normalized fluorescence imaging systems are in clinical phase for open-air intraoperative testing for bladder, ovarian, breast, and cervical cancers and melanoma $(6,10)$. In addition, multiple first-in-humans clinical trials to remove tumors using fluorescent labels for real-time, 


\section{The fluorescent effect}

A
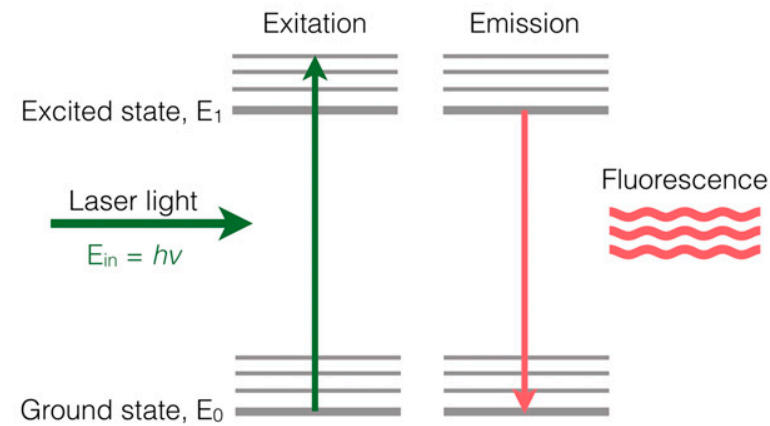

The imaging setup
B
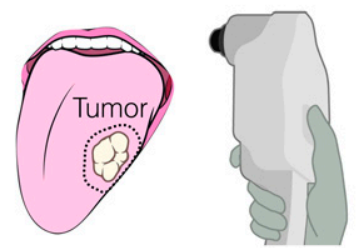

C

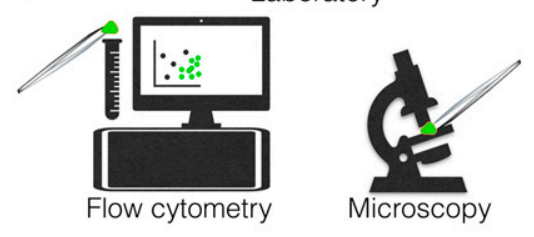

D

LASER:

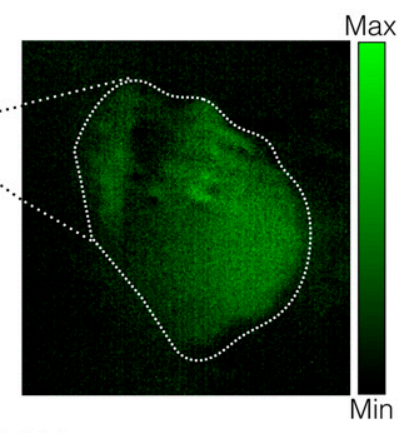

Ex/Em 488/530 nm

Light filter

$\sim 200 \mu \mathrm{m}$ depth detection
FIGURE 2. Fluorescence disease imaging. (A) Incident laser light can excite electron to $E_{1}$; relaxation of acquired excited state emits light known as fluorescence. $E_{\text {in }}$ is the incident photon energy, $v$ is its frequency, and $h$ is the Plank constant. (B) One potential application for fluorescence molecules targeting tumors is margin delineation of surface lesions, such as in oropharyngeal cancer. (C) Fluorescent dyes have broad application in laboratory settings, such as in flow cytometry sorting or fluorescent microscopy. (D) Example of use of PARPi-FL, a fluorescent molecule targeting poly(adenosine diphosphate ribose) polymerase 1, for tumor detection in clinic. $\mathrm{Ex} / \mathrm{Em}$ = excitation/emission. (Adapted with permission of (22).)

intraoperative cancer detection are under way-for example, antiepidermal growth factor receptor antibodies (cetuximab-IRDye800; Li-COR Biosciences) are being investigated in head and neck cancer (11), pancreatic cancer (12), and glioblastoma (13). Preclinical studies on nerve imaging are developing (14). Use expands to image-guided surgery in upper gastrointestinal cancers and colorectal cancers using visible light spectroscopy during surgery to improve surgical outcomes. Accordingly, intraoperative real-time fluorescence imaging has provided unprecedented detail during resection in a wide variety of tumor types (15). Early-phase clinical trials have produced a growing body of promising data supporting the utility of optical contrast agents in clinical settings. However, currently, most optical imaging agents struggle to get Food and Drug Administration approval. Fluorescence-guided surgery could significantly advance the standard of care (16). Examples of indocyanine green use include in diagnostics and preoperative planning, in vivo surgical guidance, and ex vivo surgical guidance (17) in the case of imaging hepatocellular carcinoma, head and neck cancers (18-20), and colorectal, ovarian, and breast cancers (21). In most cases, indocyanine green fluorescent imaging provides good sensitivity but poor specificity. Molecular contrast agents such as the fluorescent poly(adenosine diphosphate ribose) polymerase inhibitor that are specific to poly(adenosine diphosphate ribose) polymerase 1 might allow rapid and sensitive assays for early tumor detection and accurate assessment of surgical margins in cancer (22), which finds applications also in limited-resources settings (23). Current clinical trials using fluorescence-guided surgery aim to provide results in intraoperative imaging, specimen mapping, pathology correlation, and target validation in a wide variety of studies shown in biomarker expression analysis, laparoscopic NIR imaging, and sentinel lymph node detection $(24,25)$. The implementation of fluorescence imaging extends to hybrid tomographic systems such as the advanced fluorescence-mediated tomography/micro-CT, reported to provide a favorable alternative to classic PET analysis in drug research (26). Other device concepts such as the wide-field NIR fluorescence molecular endoscopy have been advocated for improving early detection in esophageal lesions and colorectal adenomas $(27,28)$.

Furthermore, it has been proven in the clinic that hardware upgrade improvements and the use of NIR-I and NIR-II windows for fluorescence imaging are useful in small tumors that are difficult to find during surgery and can further enhance image-guided surgery (Figs. 2B, 2C, and 2D) (29,30). Implementing fluorescence as part of intraoperative or endoscopic systems enables physicians to identify lesions via overlay of spectral unmixed fluorescent signals that are otherwise not visible under white light. There are several main barriers to the adoption of fluorescence-guided surgery as clinical routine in the hospital, including regulatory obstacles and clinical trial deliberations.

\section{Bioluminescence Imaging (BLI)}

BLI is a preclinical optical imaging technique to visualize biologic processes in vivo but is not generally considered to have potential for use in humans. Nevertheless, BLI studies are of important translational value, as this highly sensitive technique allows noninvasive monitoring of disease-relevant processes and permits tracking of cells (31). With recent developments in the field, the importance of BLI can be expected to grow in the coming years. There is a vast and growing abundance of luciferase-expressing cell lines and transgenic animal models to study diseases and potential treatments. One of the most common applications of BLI is to monitor tumor growth and metastasis formation in orthotopic xenograft models and transgenic animal models.

BLI does not require incident radiation, circumventing concerns such as phototoxicity and absorption or scattering of the excitation light. Furthermore, the near absence of background signals or autofluorescence increases the sensitivity compared with fluorescence. On the other hand, BLI is generally limited by its low 

macroscopic (but not microscopic) imaging.

\section{Principles of BLI}

Bioluminescence is a light-producing phenomenon that occurs in many species in nature, for example, bacteria, fungi, marine organisms, and terrestrial organisms such as the firefly (32). On the oxidation of a substrate (luciferin) by an enzyme (luciferase), in some cases requiring cofactors such as adenosine triphosphate and magnesium, photons are released as the substrate returns from its electronically excited state to its ground state. In this context, luciferin and luciferase are generic terms for molecules that emit light and for oxidizing enzymes that produce bioluminescence, respectively (Fig. 3A). Through genetic engineering, it became possible to express luciferases in mammalian cells and tissues, which become bioluminescent reporters on exposure to the corresponding luciferin (e.g., by intravenous injection) in preclinical disease models. For in vivo imaging, the bioluminescent signal is detected noninvasively using charge-coupled-device cameras for light detection (Figs. 3B and 3C).

The most commonly used luciferases in BLI are firefly luciferase and click beetle luciferase. Both use d-luciferin as substrate in dependence on the cofactors adenosine triphosphate and $\mathrm{Mg}^{++}$ and produce red light with a broad emission peak at approximately $600 \mathrm{~nm}$.

\section{Technologic Advances}

Research efforts have focused on developing luciferin-luciferase systems that are shifted to the NIR $(>650 \mathrm{~nm})$, are brighter, and have faster kinetics and a more sustained signal than naturally occurring systems. brightness and low spatiotemporal resolution, which allows only

Emissions between 610 and $650 \mathrm{~nm}$ have been achieved by mutagenesis of luciferases or synthetic modifications of the substrate (33). However, in most cases, the total light output in vivo was not strongly enhanced, for reasons such as a decrease in quantum yield or reduced activity of synthetic luciferins. Another approach to red-shift emission is bioluminescence resonance energy transfer. Bioluminescence resonance energy transfer systems have shown increased quantum yield and brightness, enabling subcellular imaging or flow cytometry (34). More recently, luciferinluciferase engineering was combined with bioluminescence resonance energy transfer, creating a system, called Antares2, that emits up to 65 times more photons above $600 \mathrm{~nm}$ than firefly luciferase/dluciferin in cells and offers substantially increased signal intensity and tissue penetration in vivo (35). In vivo NIR BLI has also been enabled by the development of infra luciferin, with an emission peak up to $706 \mathrm{~nm}$ (36) and a mutated click beetle luciferase (click beetle luciferase 2) (emission, 730 and 743 $\mathrm{nm}$ ) (37), showing substantial increases in brightness, sensitivity, stability, and tissue penetration. Lastly, caged luciferin substrates have been introduced, which are conceptually comparable to activatable fluorescent probes. Caged substrates are modified with functional groups, which must be cleaved off before interaction with luciferase becomes possible-for example, by enzymatic cleavage (e.g., caspases) or by activity of a drug or microenvironment conditions (e.g., pH). Red-shifted, brighter, and more stable BLI systems could also contribute to advances in bioluminescence tomography. In bioluminescence tomography, multispectral 2-dimensional BLI data are processed in combination with volume information, such as from CT or MRI, to reconstruct a 3-dimensional map of the light source distribution in the animal. Improved models of light propagation through tissue and new reconstruction algorithms optimized for red-shifted BLI in combination with higher photon counts could improve the accuracy, quality, and resolution of bioluminescence tomography.

Another application for BLI is cell tracking. Here, its utility extends far beyond oncology, where it can be used, for example, for stem cell tracking or, more recently, chimeric antigen receptor T-cell tracking (38). Recently, BLI of small cell populations (10 cells) and even single cells was reported (39), whereas the detection sensitivity of classic D-luciferin/firefly luciferase BLI lies upward of 1,000 cells. Monitoring of infectious disease models is an important application for BLI. Luciferase-expressing bacteria, viruses, and fungi can provide information on hostpathogen interactions and real-time response to antibiotic or antiviral treatments $(40)$. The availability of bioluminescent pairs with distinct emission spectra enables multiplexing, that is, the measurement of 2 bioluminescent signals in 1 animal. Given the above, BLI is a powerful tool to enable complex functional studies in a multitude of disease models.

FIGURE 3. Bioluminescence method for disease imaging. (A) Bioluminescence emission occurs on oxidation of substrate (luciferin) by enzyme (luciferase), in some cases requiring cofactors such as adenosine triphosphate and magnesium. (B) In BLI animal models, transplanted cells or genetically modified tissues express luciferin, whereas luciferase is delivered systemically to induce bioluminescence. (C) In small animals, bioluminescence detection usually occurs using chargecoupled-device cameras, which are suitable for low-light detection. NIR = near infrared.

\section{OPTOACOUSTIC IMAGING}

Optoacoustic imaging technology offers a new window for biomedical imaging using 


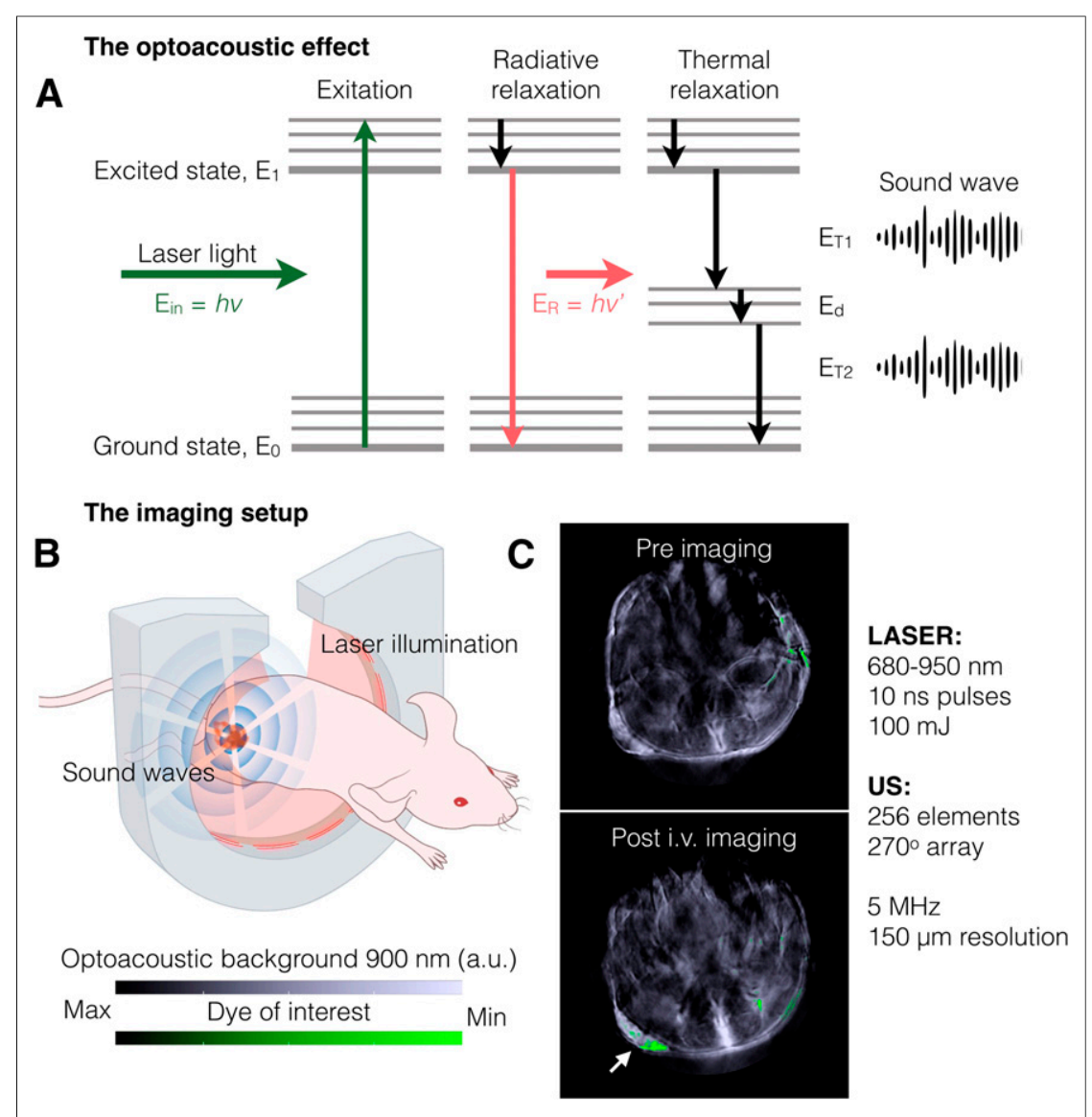

FIGURE 4. Optoacoustic methods for cancer detection. (A) Excitation from light absorption causes absorber to undergo radiative relaxation, which generates local heating. Thermal relaxation generates pressure waves and, in turn, thermoelastic expansion, known as photoacoustic effect. (B) Example of optoacoustic imaging setup (multispectral optoacoustic tomography, or MSOT), which surrounds tissue of interest with ring laser and ultrasound transducer in $270^{\circ}$ array. MSOT has tunable laser $(680-900 \mathrm{~nm})$ and allows for multispectral unmixing. (C) Representative MSOT images after multispectral unmixing before (top) and after (bottom) intravenous injection of NIR dye (green) and overlaid with optoacoustic background $(900 \mathrm{~nm}) . \mathrm{E}_{\text {in }}$ is the incident photon energy, $E_{R}$ is the relaxation energy, $E_{T 1}$ and $E_{T 2}$ represent thermal relaxation energy, and $E_{d}$ is the difference of relaxation energy. $v$ and $v^{\prime}$ refer to the photon frequency, and $h$ represents the Plank constant. a.u. $=$ arbitrary units; i.v. $=$ intravenous; $U S=$ ultrasound. (Adapted with permission of $(41,90)$.)

a combination of light for excitation and sound for detection. Sound waves scatter less than photons and thereby overcome the traditional depth limitation of optical imaging. Advanced techniques in spectral unmixing using multispectral optoacoustic tomography (MSOT), and multifrequency band splitting using the raster-scan optoacoustic mesoscopy (RSOM), have generated unprecedented detail in diseaserelated imaging, affording high-spatiotemporal and high-resolution optoacoustic imaging for cellular, tissue, and whole-body resolution (41-43). The advent of commercially available optoacoustic systems has made available a wide variety of preclinical applications ranging from neuroscience and molecular imaging of cancer to optoacoustic imaging of tumor responses to vascular-targeted therapies (44-46), which is rapidly expanding into the clinical space.

\section{Principles of Optoacoustic Imaging}

In optoacoustic imaging, agents of interest absorb light, which causes molecular vibration and small pressure waves that ultimately generate thermoelastic expansion. The resulting acoustic waves are detected by sensitive ultrasound transducers, ensuring that the acoustic coupling is achieved by water or gel medium; a variety of backprojection algorithms are used to reconstruct optoacoustic images (Fig. 4A) $(47,48)$. Many molecules can be used as contrast agents (sonophores) for optoacoustic imaging, with one of their main features being that they absorb light efficiently. These molecules can have either intrinsic or extrinsic provenance. Intrinsic includes the imaging of lipids, water, hemoglobin, fat, melanin, and collagen $(49,50)$. Since optical absorption coefficients are sensitive to wavelength, each sonophore can be extracted through spectroscopic inversion; functional information such as the abundance and location of oxyhemoglobin and deoxyhemoglobin can also be extracted. Although optoacoustic imaging has often relied on contrast-free methods, molecularly specific readout requires the use of sonophores. There are a wide variety of sonophores reported in the literature, ranging from small molecules to peptide-targeting and nanoparticles (51). The ideal sonophore should generate a signal orthogonal to and not overlapping the endogenous signal. To overcome endogenous signals, several factors must be considered, including an exogenous probe's photophysical properties (high molar extinction coefficient, sharp peak, peak absorbance in the NIR window, high photostability, low quantum yield) and biologic properties (adsorption, absorption, active targeting) (51). In addition, imaging in the NIR window can achieve a penetration depth of several millimeters with a resolution on the order of a few hundred micrometers (52). Several studies suggest that imaging agents that do not disseminate their absorbed energy through photon emission, such as dark quenchers, could advantageously generate larger orthogonal optoacoustic signals $(41,53,54)$. Reconstruction approaches and spectral unmixing methods are the key processing steps for obtaining optoacoustic images, and both heavily dictate image performance and quantification. Most 2- and 3-dimensional optoacoustic image reconstructions are performed using one of the following: closed-form domain (backprojection) solutions, closedform frequency-domain solutions, numeric time-reversal techniques, and numeric model-based algorithms $(41,47,55,56)$. Multifrequency reconstructions, splitting the high and low frequencies, improve the translational potential of this technology by allowing it to discriminate between large and small structures.

\section{Clinical Advances}

Major advances in laser technology, image reconstruction, multispectral algorithms, and inversion techniques have led to crucial improvements in optoacoustic systems $(47,57-60)$. This is especially true with the introduction of multiwavelength excitations in the NIR-1 (680-970 nm) and NIR-II (1,200-2,000 nm) lasers, allowing the advantage of imaging in the region away from high blood absorptions 


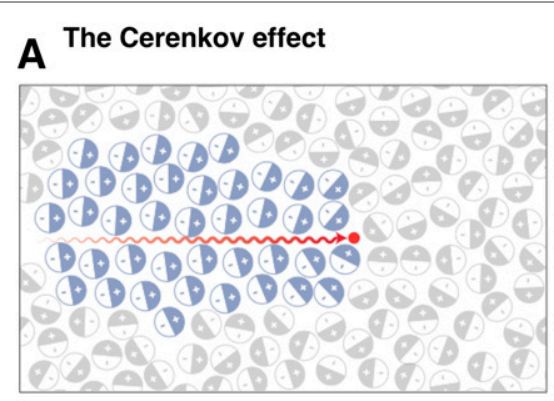

The imaging setup

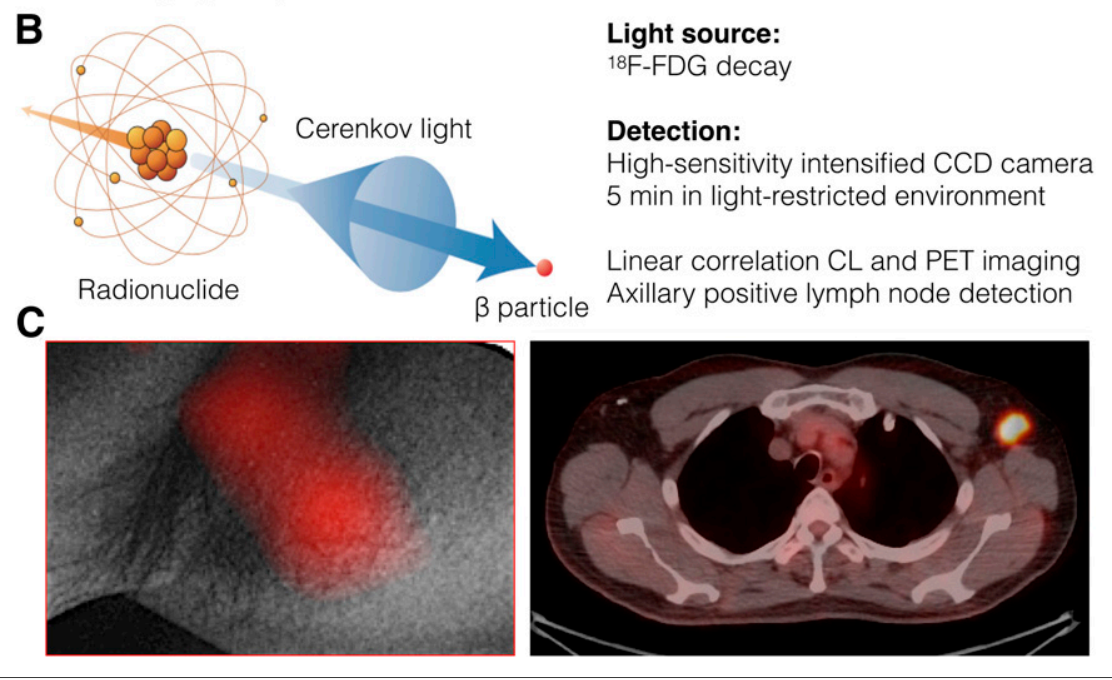

FIGURE 5. Cerenkov luminescence light for disease detection. (A) Left: Charged particle (red dot) traveling more quickly than light in medium polarizes medium. Right: As medium returns to ground state, blue-weighted light is emitted in forward direction. (B) Cerenkov light is emitted (blue cone and arrow) by medium in which charged particle travels. Radionuclides that emit $\beta$-particles with energies greater than Cerenkov threshold (261 keV in water) result in CL. (C) Left: White-light photograph from left axilla, overlaid with significant $\mathrm{CL}$ signal. Right: This signal colocalized with $\mathrm{PET} / \mathrm{CT}$ finding. $\mathrm{CCD}=$ charge-coupled device. (Adapted with permission of $(68,83)$.)

through a dielectric medium more quickly than the speed of light in that medium (Fig. 5A) (69). The molecules of the medium are randomly oriented, but they align through atom polarization induced by the charged particles passing in the vicinity; this creates a coherent wavefront described by the Huygens principle. This wavefront emits detectable photons in the same direction as the $\beta$-particle when the medium returns to its relaxed status (68). For CL, image resolution was reported to be weaker for longer wavelengths than shorter ones with increasing source depth, introducing a tradeoff between sensitivity and resolution when varying the wavelength or source depth in vivo. Preclinical studies proved it is possible to image prostate-specific membrane antigen using an ${ }^{89} \mathrm{Zr}$-labeled antibody allowing multimodal imaging, opening the doors to CL multimodal imaging with a large set of medical-use radioisotopes $(70,71)$.

\section{Clinical Advances}

Since the proof-of-concept clinical use of CL, multiple strategies of application have been investigated, from intradermal injection (72) to clinical surgery guidance (73) and margin assessment (74). CL applications have been expanded to CL-monitored gene expression (75), high-throughput screening of imaging agents $(76,77)$, theranostic applications $(78,79)$, and nanoparticle applications $(68,80-82)$. Advantages of being able to monitor clinical radiotracers in the optical spectrum include cost-effective detection and the ability to quantitatively image radionuclides that are otherwise difficult

(61). Optoacoustic imaging is being integrated into the standard of care for imaging muscular dystrophy (62), Crohn disease (63), breast cancers $(64,65)$, and skin cancers (66). Improvements in the configurations of the 2- and 3-dimensional handheld optoacoustic imaging devices have facilitated clinical translations (67). In the near future, particular focus on molecularly targeted imaging and NIR imaging system configurations performed on human and human specimens will further drive its implementation in the clinic.

\section{IMAGING}

$\mathrm{CL}$ is an optical phenomenon known to be produced by a highenergy charged particle, such as positrons or electrons $\left(\beta^{+}\right.$and $\beta^{-}$, respectively), traveling through a medium. This has been typically observed in nuclear reactors as a blue glow in the water-cooling system, and it has been reported to produce light flashes in the eyes of astronauts during space visits due to space radiation. Cerenkov light principles have been described thoroughly in a recent review article (68).

\section{Principles of CL Imaging}

$\mathrm{CL}$ is observable within the ultraviolet-to-visible spectrum and is generated when charged particles, typically $\beta$-radiation, travel to image, including ${ }^{225} \mathrm{Ac}$ and ${ }^{90} \mathrm{Y}$. CL imaging has its limitations, however, and progress in this field and in imaging instrumentation has been introduced only slowly, though preclinical studies have shown its potential using a wide range of isotopes, including ${ }^{18} \mathrm{~F}$, ${ }^{64} \mathrm{Cu},{ }^{89} \mathrm{Zr},{ }^{68} \mathrm{Ga},{ }^{124} \mathrm{I},{ }^{131} \mathrm{I}$, and ${ }^{177} \mathrm{Lu}$ (Figs. 5B, 5C, 5D) $(83,84)$. Nonradioactive $\mathrm{CL}$ imaging can be implemented using external beams to produce $\mathrm{CL}$ in tissue. A linear accelerator or a proton beam can be used as a focused source of Cerenkov photons independently of any radionuclide administration $(85,86)$, broadening the spectrum of applications of this technique.

\section{CONCLUSION}

Since the 20th century, optical imaging has developed rapidly and significantly. Pioneers from a wide range of disciplines, including physicists, engineers, mathematicians, chemists, biologists, and physicians, have made important contributions. Perhaps more so than in any other field, strong collaboration between multidisciplinary scientists could lead to unexpected, innovative approaches and new technologies. In the present review, we have summarized the main methods of nonnuclear molecular imaging as well as how and when to choose between them. Especially in the last decade, techniques for a large variety of diseases have 
TABLE 1

Comparison of Optical Imaging Modalities

\begin{tabular}{|c|c|c|c|c|}
\hline Parameter & Fluorescence & Bioluminescence & Optoacoustic & $\mathrm{CL}$ \\
\hline Principle & $\begin{array}{l}\text { Absorption of light } \\
\text { excites status of dye } \\
\text { and its relaxation } \\
\text { emits light }\end{array}$ & $\begin{array}{l}\text { Oxidation of substrate by } \\
\text { enzyme emits light }\end{array}$ & $\begin{array}{l}\text { Light is absorbed and } \\
\text { causes molecular } \\
\text { vibrations that emit } \\
\text { sound waves }\end{array}$ & $\begin{array}{l}\text { Charged particles } \\
\text { travel quickly through } \\
\text { medium and emit } \\
\text { light }\end{array}$ \\
\hline Advantage & $\begin{array}{l}\text { Easy to perform; large } \\
\text { variety of dyes }\end{array}$ & $\begin{array}{l}\text { No incident radiation } \\
\text { needed; no background }\end{array}$ & Safe & Safe and informative \\
\hline Disadvantage & $\begin{array}{l}\text { Needs excitation } \\
\text { source }\end{array}$ & Is not yet clinical & $\begin{array}{l}\text { Needs complex image } \\
\text { reconstruction }\end{array}$ & $\begin{array}{l}\text { Needs radioactive } \\
\text { preinjection }\end{array}$ \\
\hline Translatable? & Yes & Not currently & Yes & Yes \\
\hline Ease to implement & Medium & Medium & Medium & Difficult \\
\hline Ease to use & Easy & Medium & Medium & Medium \\
\hline Ease to detect & Easy & Easy & Medium & Medium \\
\hline Detectable range & $\begin{array}{l}\text { Visible spectrum and } \\
\text { NIR }\end{array}$ & $470-750 \mathrm{~nm}$ & $\begin{array}{l}400-800 \mathrm{~nm} \text { (RSOM), } \\
680-980 \mathrm{~nm} \text { (MSOT) }\end{array}$ & $\begin{array}{l}\text { Ultraviolet-to-visible } \\
\text { spectrum }\end{array}$ \\
\hline Preclinical applications & $\begin{array}{l}\text { In vitro and in vivo } \\
\text { molecular imaging }\end{array}$ & $\begin{array}{l}\text { In vitro and in vivo } \\
\text { molecular imaging }\end{array}$ & $\begin{array}{l}\text { In vivo tumor and } \\
\text { vasculature imaging }\end{array}$ & In vivo tumor imaging \\
\hline $\begin{array}{l}\text { Potential clinical } \\
\text { applications }\end{array}$ & $\begin{array}{l}\text { Screening, diagnostic, } \\
\text { intraoperative }\end{array}$ & $\begin{array}{l}\text { Stem cell or chimeric } \\
\text { antigen receptor T-cell } \\
\text { tracking }\end{array}$ & $\begin{array}{l}\text { Muscular dystrophy, } \\
\text { vasculature, cancer } \\
\text { imaging }\end{array}$ & $\begin{array}{l}\text { Positive lymph nodes } \\
\text { and cancer detection }\end{array}$ \\
\hline Cost & Low & Low & Medium & High \\
\hline
\end{tabular}

made the transition into clinical evaluation and translation, whereas others have undergone technologic advances revolutionizing their already established use. Here, we have presented principles and applications of the main optical imaging modalities. Each modality has its advantages and disadvantages. Table 1 provides a concise recap of the characteristics of each methodology. New methods are arising as well, including, for example, Raman imaging with contrast agents $(87,88)$ or the extremely elegant and interesting application of ultrasound recently found in mammalian acoustic reporting genes (89). Biologic, chemical, and mechanical engineering will lead to further technologic improvements and new applications, whereas ongoing clinical studies will consolidate the clinical value of each respective methodology. In combination, multimodal applications can augment the information gained in a single scan or observation, leading to a more complex biologic and functional understanding of the disease and therefore to improved diagnostic and treatment approaches based on the molecular makeup of a disease.

In the beginning of the era of personalized medicine, it is crucial to understand the methods of molecular imaging on which patient stratification will be based. A deep knowledge of these techniques can significantly speed up laboratory practice and, ultimately, improve patient care.

\section{REFERENCES}

1. James ML, Gambhir SS. A molecular imaging primer: modalities, imaging agents, and applications. Physiol Rev. 2012;92:897-965.

2. Lichtman JW, Conchello JA. Fluorescence microscopy. Nat Methods. 2005;2: 910-919.

3. Pierce DW, Hom-Booher N, Vale RD. Imaging individual green fluorescent proteins. Nature. 1997;388:338.

4. Rizk MA, El-Sayed SAE, AbouLaila M, et al. Performance and consistency of a fluorescence-based high-throughput screening assay for use in Babesia drug screening in mice. Sci Rep. 2017;7:12774.
5. Willmann JK, van Bruggen N, Dinkelborg LM, et al. Molecular imaging in drug development. Nat Rev Drug Discov. 2008;7:591-607.

6. Nagaya T, Nakamura YA, Choyke PL, et al. Fluorescence-guided surgery. Front Oncol. 2017;7:314.

7. Stuker F, Ripoll J, Rudin M. Fluorescence molecular tomography: principles and potential for pharmaceutical research. Pharmaceutics. 2011;3:229-274.

8. Koch M, Ntziachristos V. Advancing surgical vision with fluorescence imaging. Annu Rev Med. 2016;67:153-164.

9. Kremers G-J, Gilbert SG, Cranfill PJ, et al. Fluorescent proteins at a glance. J Cell Sci. 2011;124:157.

10. Zhang RR, Schroeder AB, Grudzinski JJ, et al. Beyond the margins: real-time detection of cancer using targeted fluorophores. Nat Rev Clin Oncol. 2017;14: 347-364.

11. Rosenthal EL, Moore LS, Tipirneni K, et al. Sensitivity and specificity of cetuximab-IRDye800CW to identify regional metastatic disease in head and neck cancer. Clin Cancer Res. 2017;23:4744-4752.

12. Tummers WS, Miller SE, Teraphongphom NT, et al. Intraoperative pancreatic cancer detection using tumor-specific multimodality molecular imaging. Ann Surg Oncol. 2018;25:1880-1888.

13. Miller SE, Tummers WS, Teraphongphom N, et al. First-in-human intraoperative near-infrared fluorescence imaging of glioblastoma using cetuximab-IRDye 800 . J Neurooncol. 2018;139:135-143.

14. Gonzales J, Demetrio de Souza Franca P, Jiang Y, et al. Fluorescence imaging of peripheral nerves by a Nav1.7-targeted inhibitor cystine knot peptide. Bioconjugate Chem. 2019;30:2879-2888.

15. van Keulen S, Nishio N, Fakurnejad S, et al. The clinical application of fluorescence-guided surgery in head and neck cancer. J Nucl Med. 2019;60:758-763.

16. Garland M, Yim JJ, Bogyo M. A bright future for precision medicine: advances in fluorescent chemical probe design and their clinical application. Cell Chem Biol. 2016;23:122-136.

17. Hu Z, Fang C, Li B, et al. First-in-human liver-tumour surgery guided by multispectral fluorescence imaging in the visible and near-infrared-I/II windows. Nat Biomed Eng. 2020;4:259-271.

18. Stubbs VC, Jaffe S, Rajasekaran K, et al. Intraoperative imaging with second window indocyanine green for head and neck lesions and regional metastasis. Otolaryngol Head Neck Surg. 2019;161:539-542.

19. Pop CF, Veys I, Gomez Galdon M, et al. Ex vivo indocyanine green fluorescence imaging for the detection of lymph node involvement in advanced-stage ovarian cancer. J Surg Oncol. 2018;118:1163-1169. 
20. Pruimboom T, van Kuijk SMJ, Qiu SS, et al. Optimizing indocyanine green fluorescence angiography in reconstructive flap surgery: a systematic review and ex vivo experiments. Surg Innov. 2020;27:103-119.

21. Ntziachristos V, Yodh AG, Schnall M, et al. Concurrent MRI and diffuse optical tomography of breast after indocyanine green enhancement. Proc Natl Acad Sci USA. 2000;97:2767.

22. Kossatz S, Pirovano G, Demétrio De Souza França P, et al. Validation of the use of a fluorescent PARP1 inhibitor for the detection of oral, oropharyngeal and oesophageal epithelial cancers. Nat Biomed Eng. 2020;4:272-285.

23. Zhu W, Pirovano G, O'Neal PK, et al. Smartphone epifluorescence microscopy for cellular imaging of fresh tissue in low-resource settings. Biomed Opt Express. 2019;11:89-98.

24. van den Bos J, Schols RM, Luyer MD, et al. Near-infrared fluorescence cholangiography assisted laparoscopic cholecystectomy versus conventional laparoscopic cholecystectomy (FALCON trial): study protocol for a multicentre randomised controlled trial. BMJ Open. 2016;6:e011668.

25. Golijanin J, Amin A, Moshnikova A, et al. Targeted imaging of urothelium carcinoma in human bladders by an ICG pHLIP peptide ex vivo. Proc Natl Acad Sci USA. 2016;113:11829-11834.

26. Hage C, Gremse F, Griessinger CM, et al. Comparison of the accuracy of FMT/ $\mathrm{CT}$ and PET/MRI for the assessment of antibody biodistribution in squamous cell carcinoma xenografts. J Nucl Med. 2018;59:44-50.

27. Hartmans E, Tjalma JJJ, Linssen MD, et al. Potential red-flag identification of colorectal adenomas with wide-field fluorescence molecular endoscopy. Theranostics. 2018;8:1458-1467.

28. Burggraaf J, Kamerling IM, Gordon PB, et al. Detection of colorectal polyps in humans using an intravenously administered fluorescent peptide targeted against c-Met. Nat Med. 2015;21:955-961.

29. Gioux S, Choi HS, Frangioni JV. Image-guided surgery using invisible nearinfrared light: fundamentals of clinical translation. Mol Imaging. 2010;9:237255.

30. Liberale G, Bourgeois P, Larsimont D, et al. Indocyanine green fluorescenceguided surgery after IV injection in metastatic colorectal cancer: a systematic review. Eur J Surg Oncol. 2017;43:1656-1667.

31. Badr CE. Bioluminescence imaging: basics and practical limitations. Methods Mol Biol. 2014;1098:1-18.

32. $\mathrm{Xu} \mathrm{T}$, Close $\mathrm{D}$, Handagama $\mathrm{W}$, et al. The expanding toolbox of in vivo bioluminescent imaging. Front Oncol. 2016;6:150.

33. Adams ST, Miller SC. Beyond D-luciferin: expanding the scope of bioluminescence imaging in vivo. Curr Opin Chem Biol. 2014;21:112-120.

34. Schaub FX, Reza MS, Flaveny CA, et al. Fluorophore-NanoLuc BRET reporters enable sensitive in vivo optical imaging and flow cytometry for monitoring tumorigenesis. Cancer Res. 2015;75:5023-5033.

35. Yeh HW, Karmach O, Ji A, et al. Red-shifted luciferase-luciferin pairs for enhanced bioluminescence imaging. Nat Methods. 2017;14:971-974.

36. Jathoul AP, Grounds H, Anderson JC, et al. A dual-color far-red to near-infrared firefly luciferin analogue designed for multiparametric bioluminescence imaging. Angew Chem Int Ed Engl. 2014;53:13059-13063.

37. Hall MP, Woodroofe CC, Wood MG, et al. Click beetle luciferase mutant and near infrared naphthyl-luciferins for improved bioluminescence imaging. Nat Commun. 2018;9:132.

38. Stowe CL, Burley TA, Allan H, et al. Near-infrared dual bioluminescence imaging in mouse models of cancer using infraluciferin. eLife. 2019;8:e45801.

39. Iwano S, Sugiyama M, Hama H, et al. Single-cell bioluminescence imaging of deep tissue in freely moving animals. Science. 2018;359:935-939.

40. Luker KE, Luker GD. Applications of bioluminescence imaging to antiviral research and therapy: multiple luciferase enzymes and quantitation. Antiviral Res. 2008;78:179-187.

41. Roberts S, Andreou C, Choi C, et al. Sonophore-enhanced nanoemulsions for optoacoustic imaging of cancer. Chem Sci. 2018;9:5646-5657.

42. Johnson SP, Ogunlade O, Lythgoe MF, et al. Longitudinal photoacoustic imaging of the pharmacodynamic effect of vascular targeted therapy on tumors. Clin Cancer Res. 2019;25:7436-7447.

43. Omar M, Schwarz M, Soliman D, et al. Pushing the optical imaging limits of cancer with multi-frequency-band raster-scan optoacoustic mesoscopy (RSOM). Neoplasia. 2015;17:208-214.

44. Haedicke K, Agemy L, Omar M, et al. High-resolution optoacoustic imaging of tissue responses to vascular-targeted therapies. Nat Biomed Eng. 2020;4:286297.

45. Gottschalk S, Degtyaruk O, Mc Larney B, et al. Rapid volumetric optoacoustic imaging of neural dynamics across the mouse brain. Nat Biomed Eng. 2019;3:392401.

46. Roberts S, Seeger M, Jiang Y, et al. Calcium sensor for photoacoustic imaging. J Am Chem Soc. 2018;140:2718-2721.
47. Glatz J, Deliolanis NC, Buehler A, et al. Blind source unmixing in multi-spectral optoacoustic tomography. Opt Express. 2011;19:3175-3184.

48. Tzoumas S, Ntziachristos V. Spectral unmixing techniques for optoacoustic imaging of tissue pathophysiology. Philos Trans A Math Phys. Eng Sci. 2017;375: 20170262.

49. Zhang HF, Maslov K, Stoica G, et al. Functional photoacoustic microscopy for highresolution and noninvasive in vivo imaging. Nat Biotechnol. 2006;24:848-851.

50. Pleitez MA, Khan AA, Soldà A, et al. Label-free metabolic imaging by midinfrared optoacoustic microscopy in living cells. Nat Biotechnol. 2020;38:293296.

51. Weber J, Beard PC, Bohndiek SE. Contrast agents for molecular photoacoustic imaging. Nat Methods. 2016;13:639-650.

52. Kenry, Liu B. Recent advances of optical imaging in the second near-infrared window. Adv Mater. 2018;30:e1802394.

53. Roberts S, Strome A, Choi C, et al. Acid specific dark quencher QC1 pHLIP for multi-spectral optoacoustic diagnoses of breast cancer. Sci Rep. 2019;9:8550.

54. Haedicke K, Brand C, Omar M, et al. Sonophore labeled RGD: a targeted contrast agent for optoacoustic imaging. Photoacoustics. 2017;6:1-8.

55. Lutzweiler C, Razansky D. Optoacoustic imaging and tomography: reconstruction approaches and outstanding challenges in image performance and quantification. Sensors (Basel). 2013;13:7345-7384.

56. Rosenthal A, Ntziachristos V, Razansky D. Acoustic inversion in optoacoustic tomography: a review. Curr Med Imaging Rev. 2013;9:318-336.

57. Ntziachristos V. Going deeper than microscopy: the optical imaging frontier in biology. Nat Methods. 2010;7:603-614.

58. Taruttis A, Ntziachristos V. Advances in real-time multispectral optoacoustic imaging and its applications. Nat Photonics. 2015;9:219-227.

59. Taruttis A, van Dam GM, Ntziachristos V. Mesoscopic and macroscopic optoacoustic imaging of cancer. Cancer Res. 2015;75:1548-1559.

60. Tzoumas S, Nunes A, Olefir I, et al. Eigenspectra optoacoustic tomography achieves quantitative blood oxygenation imaging deep in tissues. Nat Commun. 2016;7:12121.

61. Upputuri PK, Pramanik M. Photoacoustic imaging in the second near-infrared window: a review. J Biomed Opt. 2019;24:1-20.

62. Regensburger AP, Fonteyne LM, Jüngert J, et al. Detection of collagens by multispectral optoacoustic tomography as an imaging biomarker for Duchenne muscular dystrophy. Nat Med. 2019;25:1905-1915.

63. Knieling F, Neufert C, Hartmann A, et al. Multispectral optoacoustic tomography for assessment of Crohn's disease activity. N Engl J Med. 2017;376:1292-1294.

64. Becker A, Masthoff M, Claussen J, et al. Multispectral optoacoustic tomography of the human breast: characterisation of healthy tissue and malignant lesions using a hybrid ultrasound-optoacoustic approach. Eur Radiol. 2018;28:602-609.

65. Heijblom M, Piras D, van den Engh FM, et al. The state of the art in breast imaging using the Twente Photoacoustic Mammoscope: results from 31 measurements on malignancies. Eur Radiol. 2016;26:3874-3887.

66. Chen Z, Rank E, Meiburger KM, et al. Non-invasive multimodal optical coherence and photoacoustic tomography for human skin imaging. Sci Rep. 2017; 7:17975.

67. Neuschmelting V, Burton NC, Lockau H, et al. Performance of a multispectral optoacoustic tomography (MSOT) system equipped with $2 \mathrm{D}$ vs. 3D handheld probes for potential clinical translation. Photoacoustics. 2015;4:1-10.

68. Shaffer TM, Pratt EC, Grimm J. Utilizing the power of Cerenkov light with nanotechnology. Nat Nanotechnol. 2017;12:106-117.

69. Das $\mathrm{S}$, Thorek DLJ, Grimm J. Cerenkov imaging advances in cancer research. In: Pomper MG, Fisher PB, eds. Advances in Cancer Research. Vol. 124. Cambridge, MA: Academic Press; 2014:213-234.

70. Ruggiero A, Holland JP, Lewis JS, et al. Cerenkov luminescence imaging of medical isotopes. J Nucl Med. 2010;51:1123-1130.

71. Spinelli AE, Ferdeghini M, Cavedon C, et al. First human Cerenkography. $J$ Biomed Opt. 2013;18:20502.

72. Thorek DL, Abou DS, Beattie BJ, et al. Positron lymphography: multimodal, high-resolution, dynamic mapping and resection of lymph nodes after intradermal injection of ${ }^{18}$ F-FDG. J Nucl Med. 2012;53:1438-1445.

73. Kothapalli SR, Liu H, Liao JC, et al. Endoscopic imaging of Cerenkov luminescence. Biomed Opt Express. 2012;3:1215-1225.

74. Grootendorst MR, Cariati M, Pinder SE, et al. Intraoperative assessment of tumor resection margins in breast-conserving surgery using ${ }^{18} \mathrm{~F}-\mathrm{FDG}$ Cerenkov luminescence imaging: a first-in-human feasibility study. J Nucl Med. 2017;58:891898.

75. Liu H, Ren G, Liu S, et al. Optical imaging of reporter gene expression using a positron-emission-tomography probe. J Biomed Opt. 2010;15:060505.

76. Fan D, Zhang X, Zhong L, et al. ${ }^{68} \mathrm{Ga}$-labeled 3PRGD2 for dual PET and Cerenkov luminescence imaging of orthotopic human glioblastoma. Bioconjug Chem. 2015;26:1054-1060. 
77. Robertson R, Germanos MS, Manfredi MG, et al. Multimodal imaging with ${ }^{18} \mathrm{~F}-$ FDG PET and Cerenkov luminescence imaging after MLN4924 treatment in a human lymphoma xenograft model. J Nucl Med. 2011;52:1764-1769.

78. Pandya DN, Hantgan R, Budzevich MM, et al. Preliminary therapy evaluation of ${ }^{225}$ Ac-DOTA-c(RGDyK) demonstrates that Cerenkov radiation derived from ${ }^{225}$ Ac daughter decay can be detected by optical imaging for in vivo tumor visualization. Theranostics. 2016;6:698-709.

79. Balkin ER, Kenoyer A, Orozco JJ, et al. In vivo localization of ${ }^{90} \mathrm{Y}$ and ${ }^{177} \mathrm{Lu}$ radioimmunoconjugates using Cerenkov luminescence imaging in a disseminated murine leukemia model. Cancer Res. 2014;74:5846-5854.

80. Black KC, Ibricevic A, Gunsten SP, et al. In vivo fate tracking of degradable nanoparticles for lung gene transfer using PET and Ĉerenkov imaging. Biomaterials. 2016;98:53-63.

81. Guo W, Sun $\mathrm{X}$, Jacobson $\mathrm{O}$, et al. Intrinsically radioactive $\left[{ }^{64} \mathrm{Cu}\right] \mathrm{CuInS} / \mathrm{ZnS}$ quantum dots for PET and optical imaging: improved radiochemical stability and controllable Cerenkov luminescence. ACS Nano. 2015;9:488-495.

82. Lee SB, Yoon G, Lee SW, et al. Combined positron emission tomography and Cerenkov luminescence imaging of sentinel lymph nodes using PEGylated radionuclide-embedded gold nanoparticles. Small. 2016;12:4894-4901.
83. Thorek DL, Riedl CC, Grimm J. Clinical Cerenkov luminescence imaging of ${ }^{18}$ F-FDG. J Nucl Med. 2014;55:95-98.

84. Tamura R, Pratt EC, Grimm J. Innovations in nuclear imaging instrumentation: Cerenkov imaging. Semin Nucl Med. 2018;48:359-366.

85. Zhang R, D'souza AV, Gunn JR, et al. Cherenkov-excited luminescence scanned imaging. Opt Lett. 2015;40:827-830.

86. Helo Y, Kacperek A, Rosenberg I, et al. The physics of Cerenkov light production during proton therapy. Phys Med Biol. 2014;59:7107-7123.

87. Nicolson F, Andreiuk B, Andreou C, et al. Non-invasive in vivo imaging of cancer using surface-enhanced spatially offset Raman spectroscopy (SESORS). Theranostics. 2019;9:5899-5913.

88. Andreou C, Kishore SA, Kircher MF. Surface-enhanced Raman spectroscopy: a new modality for cancer imaging. J Nucl Med. 2015;56:1295-1299.

89. Farhadi A, Ho GH, Sawyer DP, et al. Ultrasound imaging of gene expression in mammalian cells. Science. 2019;365:1469-1475.

90. Roberts S, Khera E, Choi C, et al. Optoacoustic imaging of GLP-1 receptor with a near-infrared exendin-4 analog. bioRxiv website. https://www.biorxiv.org/ content/10.1101/2020.04.29.068619v1. Published April 30, 2020. Accessed August $12,2020$. 